ACTA AGROBOTANICA

Vol. 61 (2): 239-249

2008

\title{
PLANT COMMUNITIES AND ASSOCIATIONS OF ROOT CROPS OF THE KALUSZYŃSKA UPLAND
}

\author{
Teresa Skrajna, Janina Skrzyczyńska
}

\author{
Department of Agricultural Ecology, University of Podlasie in Siedlce, B. Prusa 14, 08-110 Siedlce, Poland \\ e-mail: ekolorol@ap.siedlce.pl
}

Received: 31.05 .2007

S u m m a r y

A phytosociological classification and the characteristics of weed communities developing in root crops of the Kałuszyńska Upland are given in this paper. The presence of three associations: Digitarietum ischaemi, Echinochloo-Setarietum and Lamio-Veronicetum politae, as well as of two communities: Setaria pumila-Setaria viridis and Panico-Setarion alliance, has been found. The differentiation in trophic and soil moisture conditions of habitats results in the floristic diversity of communities, divided into lower phytosociological units. Two variants have been distinguished within the association Digitarietum ischaemi, whereas Echinochloo-Setarietum has been divided into 2 subassociations and 6 variants.

Key words: root crops, weed communities, Kałuszyńska Upland

\section{INTRODUCTION}

Plant communities accompanying root crops are characterised by a specific botanical composition which is formed primarily from the time of the completion of culturing until the harvest of the crop plant (K a p e l u s zn y, 1979; W n u k, 1989). The composition and structure of these phytocenoses depend on many natural, agricultural practice and economic factors (D o m a ń s k a and W ó j c i k, 1974; R o la et al. 1989). Species-poor communities, devoid of characteristic species and difficult to classify, develop more and more frequently (S z o t k o w s k i, 1981; W n u k, 1989; W ęgrzy n e k, 2005).

The current state and dynamics of root crop agrocenoses have been the subject of numerous floristic and phytosociological studies (W a r c h o lińs k a, 1988; K o rnia k, 1992; S krzy c zyń s ka , 1994; Kuźn i ew s ki, 1996; Wójcik, 2000; S i c iński, 2003; W ęgr z y n e k, 2005).

This publication is a continuation of the characteristics of segetal vegetation of the Kałuszyńska
Upland presented in earlier publications ( $\mathrm{Skrzy}$ czyńska and Skrajna, 2004; Skrajna and S krzyczyńska, 2005; 2006). The characteristics of the study area, the distribution of investigation areas in the field and methodological assumptions are presented in the paper of Skrzyczyńs a and Skraj n a (2004).

The purpose of the study was to demonstrate the floristic diversity of plant communities developing in roots crops of the Kałuszyńska Upland.

\section{MATERIALS AND METHODS}

This paper is devoted to phytocenoses accompanying root crops. Root crop communities are described based on 157 phytosociological relevés made using the Braun-Blanquet method (P a w ł o w s k i , 1972) at the end of August and in September in the years 19952000. The classification system and nomenclature of distinguished root crop communities follow $\mathrm{Ma}$ tuszkiew icz (2001), and species nomenclature follows Mirek et al. (2002).

\section{RESULTS}

Systematics of distinguished root crop communities and associations

Class: Stellarietea mediae R.Tx., Lohm. et Prsg, 1950 Order: Polygono-Chenopodietalia (R.Tx. et Lohm. 1950) J. Tx. 1961

Alliance: Panico-Setarion Siss. 1946

Association: Digitarietum ischaemi R. Tx. et Prsg. (1942) 1950

typical variant

variant with Bidens tripartita

Community Setaria pumila-Setaria viridis

Species-poor community with Panico-Setarion

Association: Echinochloo-Setarietum Krusem. et Vlieg. (1939) 1940 
Subassociation Echinochloo-Setarietum sperguletosum variant with Digitaria ischaemum

typical variant

variant with Juncus bufonius

Subassociation Echinochloo-Setarietum typicum

typical variant

variant with Juncus bufonius

variant with Fumaria officinalis

Alliance: Polygono-Chenopodion Siss. 1946

Association: Lamio-Veronicetum politae Kornaś 1950

Characteristics of distinguished associations and communities

Digitarietum ischaemi R. Tx. et Prsg (1942) 1950

In potato crops on sandy soils belonging to the very weak and weak rye complex and the weak cereal-fodder complex, Digitarietum ischaemi patches developed.

This association is described by 43 phytocenoses, out of which 28 represent the typical variant and 15 are characterised by the variant with Bidens tripartita (Tab. 1, col. 2, 3). Patches of the typical variant with Digitarietum ischaemi often occurred in the Kałuszyńska Upland area, whereas phytocenoses of the variant with Bidens tripartita rarely grew on potato plantations. In all the investigated patches, the dominant species was Digitaria ischaemum. Permanent components of this association, with large cover, comprised weeds specific to poor and acid habitats, such as: Rumex acetosella, Spergula arvensis, Scleranthus annuus, Anthemis arvensis and Raphanus raphanistrum. The structure of patches of the moist variant was distinguished by the presence of hygrophilous species, with Bidens tripartita, Juncus bufonius and Polygonum hydropiper occurring most abundantly among them.

Digitarietum ischaemi was floristically poor. In all the patches, a total of 75 species occurred (55 in the typical variant and 57 in the variant with Bidens tripartita). The average number of species in a relevé ranged between 14 in the typical variant patches and 19 in phytocenoses of the variant with Bidens tripartita.

\section{The community Setaria pumila - Setaria viridis}

This community developed on sandy soils classified as the weak rye and good rye complex (Tab. 1, col. 4). Patches of the community in question are rarely met in the Upland. They are described based on 13 phytosociological relevés in which there was a total of 59 weed species, and the number of species in a relevé ranged between 19 and 28 (on the average, it was 23). Phytocenoses of the community concerned were characterised by a large share of differential species Setaria viridis and Setaria pumila. In addition to foxtails, aci- dophilous species Spergula arvensis, Raphanus raphanistrum, Rumex acetosella and Anthemis arvensis were noted in great numbers and with large cover. Locally, patches developed which were dominated by the mass occurrence of permanent species, such as Elymus repens, Cirsium arvense and Equisetum arvense, indicating neglect in agricultural practice. Furthermore, Chenopodium album, Stellaria media, Fallopia convolvulus, Polygonum aviculare, Viola arvensis, Centaurea cyanus and Galeopsis tetrahit were found frequently and with large cover.

\section{A species-poor community from the alliance Pani- co-Setarion Siss. 1946}

On sandy soils belonging to the weak, good rye complex as well as on sandy enclaves of the very good rye complex and the weak cereal-fodder complex, species-poor patches of this community developed, devoid of characteristic species of known root crop associations (Tab. 1 col. 5). This community is described based on 23 phytosociological relevés. It was made up of 60 species; the number of species in a relevé ranged from 14 up to 29 (on the average, 20).

The characteristic feature of the community was the numerous occurrences of acidophilous species, characteristic and distinguishing for the alliance Panico - Setarion, such as: Rumex acetosella, Spergula arvensis and Raphanus raphanistrum, as well as the absence of species characteristic for associations of this alliance. Out of species characteristic for the order Polygono-Chenopodietalia, only Chenopodium album and Stellaria media occurred more frequently and in greater numbers. Common weeds characteristic for the class Stallarietea mediae, such as: Anthemis arvensis, Fallopia convolvulus, Polygonum aviculare and Viola arvensis, comprised a large group, and from companion species, Elymus repens and Equisetum arvense occurred in great numbers.

Patches of the community in question were also found in excessively moist habitats, locally with a large share of Equisetum sylvaticum, Polygonum hydropiper and Gnaphalium uliginosum.

Echinochloo-Setarietum Krusem. et Vlieg. (1939) 1940

Echinochloo-Setarietum was a commonly occurring association of root crops in the Kałuszyńska Upland. Patches of this association developed in different trophic and soil moisture conditions. These soils were most frequently formed from sands of different origin (rarely from clays) belonging to all soil complexes occurring in the Kałuszyńska Upland, except for the weak rye complex. The wide amplitude of occupied habitats was reflected in the species composition and 
Table 1

Digitarietum ischaemi R. Tx. et Prsg. (1942) 1950, community Setaria pumila-Setaria viridis, impoverished community from alliance Panico-Setarion Siss. 1946.

\begin{tabular}{|c|c|c|c|c|c|c|c|c|}
\hline Class & \multicolumn{8}{|c|}{ Stellarietea mediae } \\
\hline Alliance & \multicolumn{8}{|c|}{ Panico-Setarion } \\
\hline Association, community & \multicolumn{4}{|c|}{ Digitarietum ischaemi } & \multicolumn{2}{|c|}{$\begin{array}{l}\text { Setaria pumila } \\
\text { - Setaria viridis }\end{array}$} & \multicolumn{2}{|c|}{$\begin{array}{c}\text { Impoverished } \\
\text { community from } \\
\text { Panico-Setarion } \\
\end{array}$} \\
\hline Variant & \multicolumn{2}{|c|}{ typical } & \multicolumn{4}{|c|}{$\begin{array}{l}\text { with Bidens } \\
\text { tripartita }\end{array}$} & & \\
\hline \multirow{5}{*}{ Soil unit } & \multicolumn{2}{|c|}{ 7.6.5 } & \multicolumn{2}{|c|}{$9,6,5$} & \multicolumn{2}{|c|}{6,5} & \multicolumn{2}{|c|}{$9,6,5,4$} \\
\hline & \multicolumn{2}{|c|}{$\mathrm{A}, \mathrm{Bw}$} & \multicolumn{2}{|c|}{ A,Bw,M } & \multicolumn{2}{|c|}{$\mathrm{A}, \mathrm{Bw}$} & \multicolumn{2}{|c|}{$\mathrm{A}, \mathrm{Bw}, \mathrm{Dz}$} \\
\hline & \multicolumn{2}{|c|}{$\mathrm{pl}$} & \multicolumn{2}{|c|}{ ps:pl } & \multicolumn{2}{|c|}{ ps.pl } & \multicolumn{2}{|c|}{ ps:pl/gl } \\
\hline & \multicolumn{2}{|c|}{ ps:pl } & \multicolumn{2}{|c|}{ pgl.gl } & \multicolumn{2}{|c|}{ pgl.gl/gs } & \multirow{2}{*}{\multicolumn{2}{|c|}{$\begin{array}{l}\text { pgl:gl/gs } \\
\text { pgm:gl }\end{array}$}} \\
\hline & \multicolumn{2}{|c|}{ pgl.pl } & \multicolumn{2}{|c|}{ pgm:gs } & & & & \\
\hline Number of phytosociological releves & & & & & & & & \\
\hline Number of species in a releve & & & & & & & & \\
\hline Ground cover by weeds in $\%$ & & & & & & & & \\
\hline Number of species & & & & & & & & \\
\hline 1 & & & & & & & & \\
\hline & $\mathrm{S}$ & $\mathrm{D}$ & $\mathrm{S}$ & $\mathrm{D}$ & $\mathrm{S}$ & $\mathrm{D}$ & $\mathrm{S}$ & $\mathrm{D}$ \\
\hline I. Ch. Digitarietum ischaemi & & & & & & & & \\
\hline Digitaria ischaemum & $\mathrm{V}$ & 1857 & $\mathrm{~V}$ & 1167 & & & & \\
\hline II. Ch.D. Panico-Setarion & & & & & & & & \\
\hline Rumex acetosella & V & 659 & $\mathrm{~V}$ & 167 & IV & 69 & IV & 219 \\
\hline Spergula arvensis & V & 412 & $\mathrm{~V}$ & 420 & IV & 454 & $\mathrm{~V}$ & 348 \\
\hline Scleranthus annuus & IV & 184 & II & 27 & II & 61 & III & 69 \\
\hline Raphanus raphanistrum & III & 57 & III & 73 & V & 408 & V & 189 \\
\hline Setaria viridis & II & 71 & II & 67 & V & 854 & & \\
\hline Setaria pumila & & & & & $\mathrm{V}$ & 788 & & \\
\hline III. Ch. D. Polygono-Chenopodietali & & & & & & & & \\
\hline Chenopodium album & III & 141 & III & 167 & IV & 354 & $\mathrm{~V}$ & 260 \\
\hline Stellaria media & II & 50 & II & 40 & IV & 242 & IV & 400 \\
\hline Matricaria maritima subsp. inodora & & & & & III & 108 & II & 61 \\
\hline Sonchus arvensis & & & & & II & 38 & III & 43 \\
\hline Polygonum tomentosum & & & & & II & 61 & II & 39 \\
\hline Capsella bursa-pastoris & & & & & & & II & 26 \\
\hline Conyza canadensis & II & 25 & & & & & & \\
\hline IV. D. var. with hygrophilous specie & & & & & & & & \\
\hline Juncus bufonius & & & $\mathrm{V}$ & 523 & & & II & 22 \\
\hline Bidens tripartita & & & $\mathrm{V}$ & 1000 & II & 59 & & \\
\hline Polygonum hydropiper & & & IV & 173 & II & 92 & III & 74 \\
\hline Mentha arvensis & & & II & 53 & II & 23 & II & 39 \\
\hline
\end{tabular}


cd. table 1

\begin{tabular}{|c|c|c|c|c|c|c|c|c|}
\hline Gnaphalium uliginosum & & & II & 67 & II & 31 & III & 56 \\
\hline Plantago intermedia & & & II & 27 & & 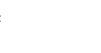 & & \\
\hline Equisetum sylvaticum & & & II & 27 & & 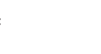 & II & 119 \\
\hline Spergularia rubra & & & & & & & II & 35 \\
\hline Gypsophila muralis & & & & & II & 31 & II & 35 \\
\hline \multicolumn{9}{|l|}{ V. Ch. Stellarietea mediae } \\
\hline Anthemis arvensis & IV & 159 & III & 127 & V & 242 & $\mathrm{~V}$ & 259 \\
\hline Fallopia convolvulus & $\mathrm{V}$ & 125 & IIII & 87 & IV & 138 & $\mathrm{~V}$ & 211 \\
\hline Polygonum aviculare & III & 57 & II & 27 & IV & 138 & $\mathrm{~V}$ & 135 \\
\hline Viola arvensis & II & 87 & IV & 100 & IV & 265 & $\mathrm{~V}$ & 139 \\
\hline Centaurea cyanus & II & 32 & \multicolumn{2}{|c|}{$*$} & IV & 138 & III & 198 \\
\hline Galeopsis tetrahit & II & 39 & III & 733 & IV & 296 & IV & 96 \\
\hline Arnoseris minima & II & 36 & II & 33 & \multicolumn{2}{|c|}{ * } & \multicolumn{2}{|c|}{$*$} \\
\hline Teesdalea nudicaulis & II & 50 & & & & & & \\
\hline Vicia hirsuta & & & II & 27 & \multicolumn{2}{|c|}{$*$} & \multicolumn{2}{|c|}{$*$} \\
\hline Myosotis arvensis & & & & & II & 38 & III & 48 \\
\hline Arabidopsis thaliana & \multirow{2}{*}{\multicolumn{2}{|c|}{$*$}} & & & \multicolumn{2}{|c|}{$*$} & & \\
\hline Vicia tetrasperma & & & & & \multicolumn{2}{|c|}{$*$} & II & 26 \\
\hline \multicolumn{9}{|l|}{ VI. Companion species } \\
\hline Elymus repens & IV & 75 & V & 167 & $\mathrm{~V}$ & 911 & $\mathrm{~V}$ & 556 \\
\hline Convolvulus arvensis & IV & 89 & II & 40 & III & 54 & II & 56 \\
\hline Achillea millefolium & IV & 71 & II & 33 & III & 54 & III & 48 \\
\hline Erodium cicutarium & II & 32 & III & 53 & \multicolumn{2}{|c|}{$*$} & II & 22 \\
\hline Equisetum arvense & II & 24 & III & 197 & $\mathrm{~V}$ & 1135 & $\mathrm{~V}$ & 554 \\
\hline Polygonum lapathifolium subsp. lapathifolium & II & 50 & II & 40 & II & 69 & III & 230 \\
\hline Cirsium arvense & \multicolumn{2}{|c|}{$*$} & II & 40 & $\mathrm{~V}$ & 769 & IV & 272 \\
\hline Polygonum persicaria & \multicolumn{2}{|c|}{$*$} & II & 33 & III & 130 & III & 170 \\
\hline Veronica arvensis & \multicolumn{2}{|c|}{$*$} & \multicolumn{2}{|c|}{$*$} & II & 23 & II & 157 \\
\hline Plantago maior & & & \multicolumn{2}{|c|}{$*$} & III & 27 & & \\
\hline Cerastium holosteoides & & & & & \multicolumn{2}{|c|}{$*$} & II & 35 \\
\hline Pоа аппиа & \multicolumn{2}{|c|}{$*$} & \multicolumn{2}{|c|}{$*$} & II & 23 & II & 48 \\
\hline Trifolium repens & \multicolumn{2}{|c|}{$*$} & \multirow{2}{*}{\multicolumn{2}{|c|}{$*$}} & III & 38 & & \\
\hline Galeopsis ladanum & \multicolumn{2}{|c|}{$*$} & & & II & 196 & & \\
\hline Erophila verna & II & 50 & & & & & & \\
\hline
\end{tabular}

Sporadic species: III - Rumex crispus 3,4; Geranium pusillum 4,5; Oxalis fontana 3; Veronica persica 5; Sonchus oleraceus 5; Lamium purpureum 5; IV - Ranunculus repens 3,4,5; Sagina procumbens 3,5; Hypericum humifusum 3,4,5; Illecebrum verticillatum 3; Juncus capitatus 3; Peplis portula 3; V - Vicia villosa 2,4; Vicia angustifolia 3,5; Papaver argemone 2; Chamomilla recutita 4; Apera spica-venti 2; Vicia sativa 4; VI - Taraxacum officinale 2,3,4,5; Artemisia vulgaris 2,4,5; Cerastium arvense 2 ,3, 4; Knautia arvensis 2,3,4,5; Trifolium arvense 2, 3; Veronica serpyllifolia 3; Leontodon autumnalis 5; Stellaria graminea 2, 4; Arenaria serpyllifolia 5; Chenopodium rubrum 2,3,5; Ornithopus sativus 2; Lupinus luteus 2; Myosotis stricta 2; Medicago lupulina 5; Plantago media 4,5; Chamomilla suaveolens 5; Poa pratensis 5; Hypochoeris glabra 4; Galeopsis pubescens 5; Rorippa palustris 5; Artemisia absinthium 2; Anthoxanthum aristatum 2,3; Pimpinella saxifraga 2; Veronica dillenii 2; Holcus mollis 2; Hieracium pilosella 2; Cerastium semidecandrum 2; Alopecurus pratensis 5; Crepis tectorum 5;

Explanatory notes: numbers after species inform about numbers of columns in the table: $\mathrm{S}$ - phytosociological constancy, D - cover factor

* - species constancy with little cover. 
Table 2

Echinochloo-Setarietum Krus. et Vlieg. (1939) 1940, Lamio-Veronicetum politeae Kornaś 1950.

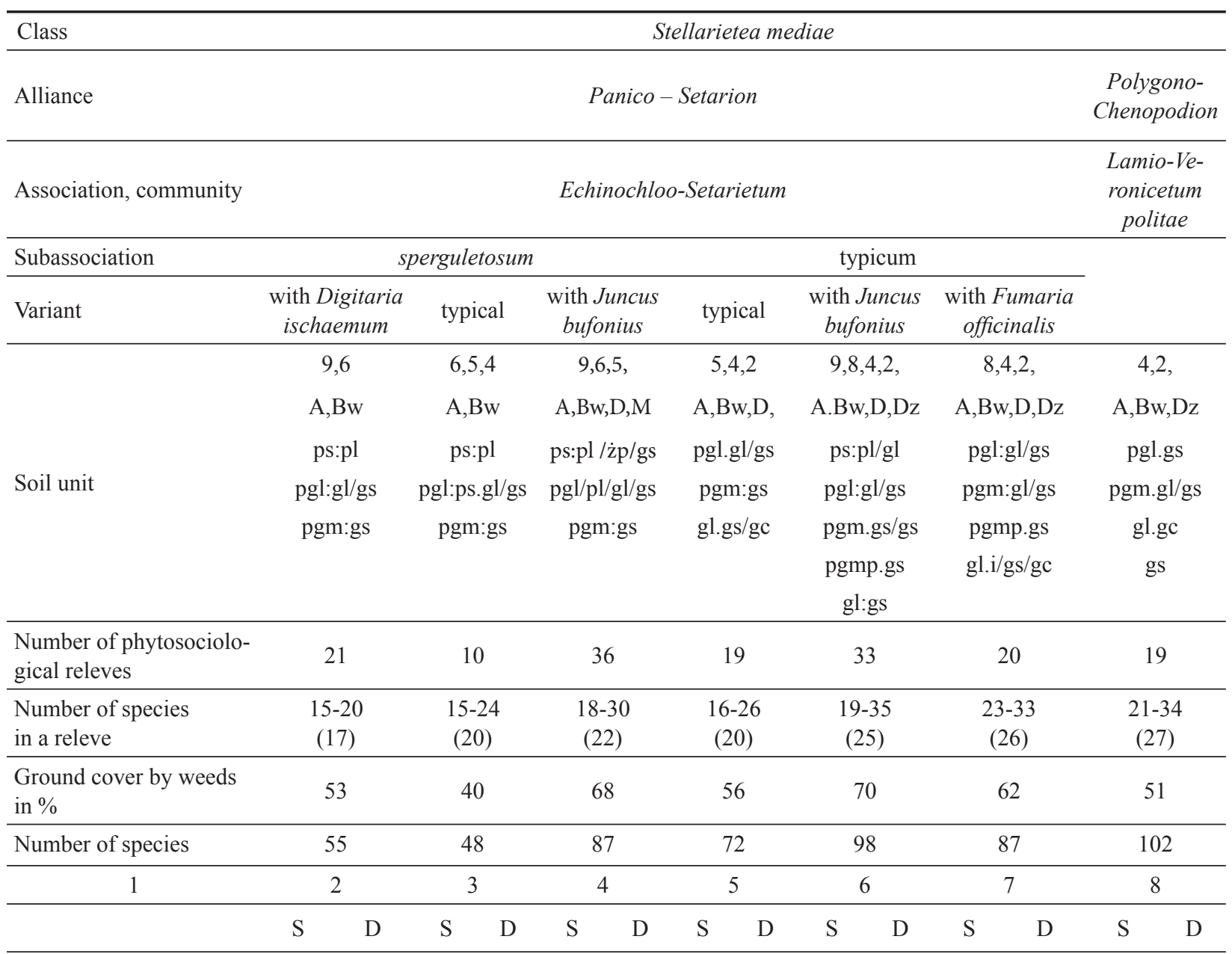

\section{Ch. D. Echinochloo-}

Setarietum

\section{Panico-Setarion}

\begin{tabular}{lcccccccccccccc} 
Digitaria ischaemum & V & 1012 & & & & & & & & & & & \\
Rumex acetosella & IV & 171 & IV & 325 & V & 278 & & & $*$ & & $*$ & & $*$ \\
Spergula arvensis & V & 691 & V & 750 & V & 1299 & $*$ & & $*$ & & & $*$ & $*$ & $*$ \\
Scleranthus annuus & II & 52 & III & 140 & III & 67 & & & & & & & $*$ \\
Raphanus raphanistrum & V & 514 & III & 100 & V & 319 & III & 58 & III & 176 & $*$ & & $*$ & $*$ \\
Setaria viridis & III & 119 & II & 70 & II & 36 & II & 74 & $*$ & & & & $*$ \\
Setaria pumila & III & 109 & II & 70 & II & 44 & III & 100 & II & 33 & II & 30 & $*$ & $*$ \\
Echinochloa crus-galli & V & 545 & V & & V & 1396 & V & 1539 & V & 1030 & V & 937 & II & 337 \\
\hline
\end{tabular}

II. D. var. with Fumaria officinalis

Fumaria officinalis

III. Ch. PolygonoChenopodion

\begin{tabular}{llllllllllllllll} 
Galinsoga parviflora & II & 202 & II & 30 & III & 194 & V & 740 & V & 921 & IV & 572 & II & 197 \\
Galinsoga ciliata & $*$ & & $*$ & & II & 96 & III & 121 & III & 685 & II & 120 & $*$ & \\
\hline
\end{tabular}


cd. table 2

\begin{tabular}{|c|c|c|c|c|c|c|c|c|c|c|c|c|c|c|}
\hline $\begin{array}{l}\text { Matricaria maritima } \\
\text { subsp. inodora }\end{array}$ & & & & & III & 53 & IV & 110 & IV & 208 & IV & 187 & IV & 84 \\
\hline Euphorbia helioscopia & & & & & & & II & 21 & II & 33 & IV & 272 & $\mathrm{~V}$ & 326 \\
\hline Veronica persica & & & & & & & II & 21 & $*$ & & II & 30 & IV & 242 \\
\hline Veronica agrestis & & & & & & & II & 32 & $*$ & & $*$ & & IV & 114 \\
\hline Sonchus oleraceus & & & & & & & $*$ & & $*$ & & III & 80 & III & 53 \\
\hline $\begin{array}{l}\text { Erysimum } \\
\text { cheiranthoides }\end{array}$ & & & & & * & & * & & $*$ & & II & 60 & III & 124 \\
\hline $\begin{array}{l}\text { Chenopodium } \\
\text { polyspermum }\end{array}$ & & & & & & & $*$ & & & & III & 173 & IV & 305 \\
\hline Solanum nigrum & & & & & & & $*$ & & & & II & 132 & II & 21 \\
\hline Lamium purpureum & & & & & * & & $*$ & & $*$ & & II & 177 & IV & 224 \\
\hline Lamium amplexicaule & & & & & & & $*$ & & $*$ & & II & 100 & $\mathrm{~V}$ & 279 \\
\hline Veronica polita & & & & & & & & & & & & & $\mathrm{V}$ & 676 \\
\hline Veronica opaca & & & & & & & & & & & & & III & 147 \\
\hline Sonchus asper & & & & & & & & & $*$ & & $*$ & & II & 42 \\
\hline Chaenorchinum minus & & & & & & & & & & & $*$ & & II & 32 \\
\hline $\begin{array}{l}\text { IV. Ch. D. Polygono- } \\
\text { Chenopodietalia }\end{array}$ & & & & & & & & & & & & & & \\
\hline Chenopodium album & $\mathrm{V}$ & 414 & $\mathrm{~V}$ & 375 & $\mathrm{~V}$ & 507 & $\mathrm{~V}$ & 674 & $\mathrm{~V}$ & 895 & $\mathrm{~V}$ & 560 & $\mathrm{~V}$ & 847 \\
\hline Stellaria media & II & 27 & II & 30 & IV & 289 & IV & 379 & $\mathrm{~V}$ & 494 & $\mathrm{~V}$ & 755 & IV & 626 \\
\hline Capsella bursa-pastoris & $*$ & & $*$ & & II & 30 & III & 42 & III & 112 & IV & 65 & III & 47 \\
\hline Sonchus arvensis & $*$ & & $*$ & & II & 39 & IV & 105 & III & 141 & III & 80 & III & 145 \\
\hline Descurainia sophia & & & & & $*$ & & $*$ & & $*$ & & $*$ & & II & 21 \\
\hline $\begin{array}{l}\text { V. D. var. with } \\
\text { hygrophilous species }\end{array}$ & & & & & & & & & & & & & & \\
\hline Juncus bufonius & II & 89 & $*$ & & $\mathrm{~V}$ & 776 & & & $\mathrm{~V}$ & 742 & III & 85 & II & 58 \\
\hline Bidens tripartita & II & 81 & & & III & 101 & & & III & 227 & $*$ & & * & \\
\hline Polygonum hydropiper & II & 67 & & & IV & 187 & & & II & 108 & $*$ & & & \\
\hline Mentha arvensis & II & 24 & & & II & 47 & & & II & 139 & $*$ & & * & \\
\hline Gnahalium uliginosum & $*$ & & & & $\mathrm{~V}$ & 455 & & & III & 57 & $*$ & & $*$ & \\
\hline Plantago intermedia & & & & & $*$ & & & & II & 36 & * & & * & \\
\hline Equisetum sylvaticum & $*$ & & & & $*$ & & & & $*$ & & * & & & \\
\hline Rorippa sylvestris & & & & & II & 33 & & & III & 42 & $*$ & & * & \\
\hline Spergularia rubra & & & & & II & 30 & & & $*$ & & $*$ & & * & \\
\hline Gypsophila muralis & & & & & II & 36 & & & $*$ & & $*$ & & $*$ & \\
\hline Stachys palustris & & & & & $*$ & & & & II & 238 & II & 235 & $*$ & \\
\hline Potentilla anserina & & & & & * & & & & II & 33 & II & 25 & * & \\
\hline $\begin{array}{l}\text { VI. Ch. Stellarietea } \\
\text { mediae }\end{array}$ & & & & & & & & & & & & & & \\
\hline Anthemis arvensis & IV & 96 & IV & 80 & $\mathrm{~V}$ & 287 & IV & 105 & III & 67 & II & 40 & III & 68 \\
\hline Fallopia convolvulus & IV & 129 & III & 140 & IV & 105 & IV & 126 & III & 120 & III & 105 & IV & 105 \\
\hline Polygonum aviculare & IV & 119 & II & 30 & III & 86 & II & 53 & II & 24 & $*$ & & II & 47 \\
\hline Viola arvensis & IV & 90 & $\mathrm{~V}$ & 140 & IV & 117 & IV & 147 & III & 85 & IV & 125 & IV & 110 \\
\hline Centaurea cyanus & IV & 71 & IV & 70 & II & 61 & II & 32 & II & 36 & II & 35 & II & 26 \\
\hline Galeopsis tetrahit & III & 90 & $*$ & & III & 129 & II & 21 & II & 164 & $*$ & & $*$ & \\
\hline
\end{tabular}


cd. table 2

\begin{tabular}{|c|c|c|c|c|c|c|c|c|c|c|c|c|c|c|}
\hline Vicia hirsuta & $*$ & & $*$ & & $*$ & & III & 47 & III & 57 & II & 35 & II & 32 \\
\hline Myosotis arvensis & $*$ & & II & 40 & $*$ & & II & 42 & II & 45 & III & 45 & II & 32 \\
\hline Arabidopsis thaliana & & & II & 70 & $*$ & & $*$ & & II & 80 & & & $*$ & \\
\hline Vicia tetrasperma & & & $*$ & & $*$ & & $*$ & & II & 21 & $*$ & & $*$ & \\
\hline Vicia angustifolia & & & $*$ & & $*$ & & $*$ & & II & 21 & $*$ & & $*$ & \\
\hline Anagallis arvensis & & & & & & & $*$ & & $*$ & & III & 115 & III & 153 \\
\hline Thlaspi arvense & & & & & & & $*$ & & $*$ & & II & 58 & III & 58 \\
\hline Sinapis arvensis & & & & & & & $*$ & & $*$ & & II & 45 & $*$ & \\
\hline \multicolumn{15}{|l|}{ VII. Companion species } \\
\hline Elymus repens & IV & 179 & IV & 70 & IV & 130 & III & 163 & IV & 198 & IV & 212 & IV & 132 \\
\hline Convolvulus arvensis & II & 38 & II & 40 & II & 42 & III & 171 & II & 24 & $*$ & & $*$ & \\
\hline Achillea millefolium & III & 52 & II & 40 & III & 58 & $*$ & & II & 30 & $*$ & & $*$ & \\
\hline Erodium cicutarium & III & 62 & III & 50 & II & 104 & III & 79 & II & 51 & \multicolumn{2}{|c|}{ * } & $*$ & \\
\hline Equisetum arvense & IV & 719 & $\mathrm{~V}$ & 335 & IV & 296 & III & 184 & III & 227 & III & 115 & III & 84 \\
\hline $\begin{array}{l}\text { Polygonum subsp. } \\
\text { lapathifolium }\end{array}$ & IV & 176 & III & 130 & III & 118 & III & 116 & IV & 112 & III & 115 & III & 121 \\
\hline Cirsium arvense & III & 48 & II & 30 & III & 135 & IV & 126 & IV & 412 & III & 157 & II & 74 \\
\hline Polygonum persicaria & III & 169 & IV & 109 & $*$ & & III & 160 & IV & 296 & III & 84 & III & 63 \\
\hline Veronica arvensis & $*$ & & II & 40 & II & 71 & II & & III & 103 & IV & 70 & III & 182 \\
\hline Plantago maior & $*$ & & $*$ & & II & 22 & $*$ & & II & 27 & $*$ & & $*$ & \\
\hline Cerastium holosteoides & & & $*$ & & II & 22 & & & $*$ & & II & 25 & $*$ & \\
\hline Taraxacum officinale & $*$ & & $*$ & & II & 33 & $*$ & & $*$ & & II & 25 & II & 26.3 \\
\hline Galium aparine & & & & & & & III & 47 & $*$ & & II & 35 & III & 232 \\
\hline Galeopsis bifida & $*$ & & $*$ & & $*$ & & II & 47 & $*$ & & $*$ & & II & 21 \\
\hline Artemisia vulgaris & $*$ & & $*$ & & $*$ & & II & 21 & $*$ & & & & $*$ & \\
\hline Poa аппиа & $*$ & & $*$ & & $*$ & & $*$ & & II & 48 & II & 30 & II & 21 \\
\hline Neslia paniculata & & & & & & & & & $*$ & & $*$ & & III & 63 \\
\hline Melandrium album & & & $*$ & & & & $*$ & & $*$ & & * & & II & 32 \\
\hline Galium spurium & & & & & & & & & & & $*$ & & II & 26 \\
\hline Galeopsis speciosa & & & & & & & & & $*$ & & $*$ & & II & 21 \\
\hline
\end{tabular}

Sporadic species: IV - Polygonum lapathifolium subsp. pallidum 2,4,5,6,7,8; Rumex crispus 4,6,7,8; Conyza canadensis 4,5,6; Geranium pusillum 2,3,6; Oxalis fontana 5; Atriplex patula 7,8; V - Ranunculus repens 2,4,6,7,8; Sagina procumbens 4,5,6,8; Illecebrum verticillatum 4,6,8; Juncus capitatus 4,7; Polygonum amphibium 6,7,8; Peplis portula 4; Myosurus minimus 4,7; Phragmites australis 6; VI - Arnoseris minima 2,3,4; Vicia villosa 4,6,8; Chamomilla recutita 2,4,6,8; Sisymbrium officinale 4,5,6,7,8; Apera spica-venti 2, 4; Vicia sativa 4,6; Melandrium noctiflorum 7,8; Geranium dissectum 5,6,8; Papaver argemone 4; Consolida regalis 6,8; Digitaria sanguinalis 3,7; Lycopsis arvensis 7,8; Teesdalea nudicaulis 2; Lactuca serriola 4; Papaver rhoeas 8; VII - Cerastium arvense 2,3,5,7,8; Galeopsis ladanum 3,4,6,7; Knautia arvensis 2,4,6; Trifolium arvense 3,4,6; Veronica serpyllifolia 4,6,7,8; Leontodon autumnalis 2,4,6,8; Senecio vulgaris 5,6,7,8; Stellaria graminea 2,6; Daucus carota 4,5,6,8; Plantago lanceolata 2,3,5,6; Arenaria serpyllifolia 4,6, 8; Ornithopus sativus 2,4; Lupinus luteus 2,4; Myosotis stricta 3,5; Medicago lupulina 7,8; Plantago media 4; Chamomilla suaveolens 5; Trifolium pratense 5,7; Poa pratensis 4; Hypochoeris glabra 8; Galeopsis pubescens 8; Amaranthus retroflexus 8; Rorippa palustris 4; Malva neglecta 4,5; Armoracia rusticana 5,6; Artemisia absinthium 6; Urtica urens 2; Avena fatua 5; Veronica triphyllos 4; Echium vulgare 5; Cichorium intybus 5; Arabis corymbiflora 4; Ranunculus sardous 4; Epilobium roseum 6; Lysimachia nummularia 6; Lathyrus pratensis 7; Lamium album 8; Anchusa officinalis 8; Tussillago farfara 8; Artemisia campesrtris 8.

Explanatory notes: numbers after species inform about numbers of columns in the table: $\mathrm{S}$ - phytosociological constancy, D - cover factor

* - species constancy with little cover. 
the structure of phytocenoses of the association. Out of the species characteristic for this association, Echinochloa crus-galli was noted in great numbers, Raphanus raphanistrum occurred frequently, but less numerously. In addition, species characteristic for the alliance Panico-Setarion and common field weeds from higher syntaxonomic units made up a large group. The floristic diversity of phytocenoses of this association finds its reflection in subassociations and variants.

On sandy soils most frequently classified as the weak rye, good rye and weak cereal-fodder complexes, patches of Echinochloo-Setarietum sperguletosum developed (Tab. 2, col. 2, 3, 4). Acidophilous species occurring in great numbers, such as: Spergula arvensis, Rumex acetosella, Anthemis arvensis, as well as frequently noted Scleranthus annuus, Setaria pumila and Setaria viridis were the species which distinguished this subassociation. In addition, Chenopodium album, Fallopia convolvulus and Viola arvensis occurred frequently and with large cover. Locally, patches dominated by Equisetum arvense developed.

The subassociation in question is described by 67 phytocenoses, out of which 21 represent the variant with Digitaria ischaemum, 10 the typical variant, and 36 patches are characterised by the variant with Juncus bufonius.

Echinochloo-Setarietum typicum phytocenoses were found on soils more cohesive than the previously mentioned subassociation, since they were formed from light and strong loamy sands and clays, most frequently classified as the very good rye, good wheat and strong cereal-fodder complexes (Tab. 2, col. 5, 6, 7). They developed in potato and sugar beet crops. Out of species characteristic for this association, Echinochloa crus-galli occurred in great numbers, whereas the acidophilous species Raphanus raphanistrum and other species characteristic for Panico - Setarion were noted with small cover. Soil fertility and water availability in these habitats affected the floristic composition of the communities. The occurrence of species with higher trophic requirements was found in them, among others: Galinsoga parviflora, Matricaria maritima subsp. inodora, Stellaria media, Polygonum lapathifolium subsp. lapathifolium, Chenopodium album, Sonchus arvensis, Anagallis arvensis and Thlaspi arvense. Locally, in the vicinity of buildings, patches with a very large share of Galinsoga parviflora and Galinsoga ciliata occurred.

The subassociation in question is demonstrated by 72 plant patches, out of which 19 represent the typical variant, 33 the variant with Juncus bufonius, and 20 the variant with Fumaria officinalis.

\section{Lamio - Veronicetum politae Kornaś 1950}

Lamio - Veronicetum politae phytocenoses developed in potato and sugar beet crops, primarily on strong loamy sands and light clays belonging to the good wheat and very good rye complexes (Tab. 2, col. 8 ). The extent of the occurrence of this association was restricted to the most fertile soils which occur in small areas within the Upland. It is described by 19 plant patches from the Upland area.

Veronica polita and Veronica opaca are its characteristic species which show exclusive attachment to this association. Other characteristic species, Lamium amplexicaule and Veronica agrestis, though they occur frequently and may have large cover, do not belong to the most faithful of its components, as they are also found in other communities, but their optimal occurrence in the study area is in the association in question.

It is the floristically richest association of root crops within the study area. In its patches, as many as 102 species were found, on the average, 27 in one relevé.

The association in question is characterised by specific physiognomy that is determined by species occurring frequently and in great numbers which have high trophic requirements: Chenopodium polyspermum, Euphorbia helioscopia, Matricaria maritima subsp. inodora, Stellaria media, Chenopodium album, Anagallis arvensis and others. It its patches, a numerous groups of hygrophilous species was noted, with low constancy and cover.

\section{DISCUSSION}

The floristic richness of agrocenoses developing in root crops of the Kałuszyńska Upland was determined by trophic and moisture conditions of habitats as well as by diverse agricultural practices. Communities of species with a narrow range of tolerance are characterised by great sensitivity. Digitarietum ischaemi, which is marked by low persistence, can be included in such associations. The intensification of chemical protection and increased fertilisation (D o m a n s k a and W ó j c i k, 1974) as well as changes in land use methods (fallowing or afforestation) result in the impoverishment of the floristic composition and the limitation in the occurrence of this association (W ę gr z y nek, 2005).

In the study area, on the poorest soils, in trophic terms, and on dry soils, potato crops were often colonised by the floristically poorest patches of Digitarietum ischaemi, but on small areas. Slightly richer phytocenoses with a share of hygrophilous species developed on light loamy sands. This Subatlantic association has better development conditions in western and northern Poland (Kornás , 1950), as reported by B orowiec and Kutyna (1989). It is also often found in certain regions of the central ( $\mathrm{S}$ i c iń s k i 
et al. 1978; Warcholińska, 1987,1998) and eastern (Skrzyczyńska, 1994; Skrajna and Skrzyczyńska, 2004) parts of the country. In many regions, Digitarietum ischaemi phytocenoses occur rarely and they significantly reduce their extent of occurrence. It applies, inter alia, to Poland's southern regions (K u źn i e w s k i, 1974; S z o t k o w s k i, 1989; Wnuk, 1989; Ani oł-Kwiatkowska, 1990; K o z a k, 2002; W ęg r z y n e k, 2005).

Setaria pumila - Setaria viridis communities are met in similarly poor habitats (rarely in the study area). Rzy m ow s k a (1999) reported from the Podlaski Przełom Bugu mesoregion phytocenoses with a floristic composition similar to that presented in this paper.

Patches devoid of characteristic species, classified as species-poor communities from the alliance $P$ anico-Setarion, were also rarely found in root crops of the study area. Such impoverished communities have been described by Szotkowski (1981) and Kuźniewski (1974) in Lower Silesia, by Wnuk (1989) in the Częstochowa Upland (Wyżyna Częstochowska), by Wnuk et al. (1989) in the Rzeszów area, and by Węgrzynek (2005) in the Silesian Upland (Wyżyna Ślaska).

Likewise across the country (W nuk, 1976, 1989; K a p e l u s z n y, 1979; W ó j c i k, 1980; K u tyna, 1988; Anioł-Kwiatkowska, 1990; Skrzyczyńska, 1994; Warcholińska, 1998; K ozak, 2002; S i ciński 2003; Węgrzynek, 2005), Echinochloo-Setarietum was the association commonly occurring in the Kałuszyńska Upland. This is a result of broad-scale ecological requirements of species characteristic for this association and nowadays of the wide occurrence of Echinochloa crus-galli biotypes resistant to the effect of herbicides ( $\mathrm{R}$ o $1 \mathrm{a}$ et al. 1989; J ę druszczak and Antoszek, 2002).

The floristically richest patches of Lamio-Veronicetum politae were found very rarely in the study area. The reason for this is the small acreage of proper habitats. This association reaches the optimum of its development on alkaline $\mathrm{pH}$ soils, on loess, rendzina and black-earth soils. Judging from literature data, a conclusion can be drawn that this association occurs widely across the country. From other regions, its has been reported by: Kornaś (1972), K a p e lus zny (1979), W ój cik (1980), S z otkow s ki (1981), Kutyna (1988), Wnuk (1989), A ni oł-K w iat kowska (1990), Hołdyński (1991), Trąba and Ziemińska (1994), Warcholińska (1994), K o zak (2002), S i c ińs ki (2003).

\section{CONCLUSIONS}

1. In root crops of the Kałuszyńska Upland, Echinochloo-Setarietum patches were noted most frequent- ly, differentiated into subassociations and variants.

2. On poor sandy soils, Digitarietum ischaemi phytocenoses occurred often, Setaria pumilla - Setaria viridis communities - less frequently.

3. The floristically rich patches of Lamio-Veronicetum politae grew in the most fertile habitats.

\section{REFERENCES}

Anioł-Kwiatkowska J., 1990. Zbiorowiska segetalne Wału Trzebnickiego. Florystyczno-ekologiczne studium porównawcze. / Segetal communities of the Trzebnica Moraine Belt. A floristic and ecological comparative study. Wyd. Univ. Wrock. Prace Bot.: 46-230.

Borowiec S., Kutyna I., 1989. Zróżnicowanie zachwaszczenia upraw na glebach lekkich północno-zachodniej Polski. / Differences in weed infestation of crops on light soils in north-western Poland. Zesz. Nauk. WSRP w Siedlcach Ser. Rolnictwo, 20: 95-102.

Domańska H., Wójcik Z., 1974. Wpływ działalności człowieka na zbiorowiska roślinne pól uprawnych (w:) Rejonizacja chwastów segetalnych dla potrzeb rolnictwa. Materiały sympozjum we Wrocławiu / The effect of human activity on plant communities of crop fields. (In): The regionalisation of segetal weeds for agricultural needs. Materials of a symposium in Wrocław. IUNG, Puławy, 82: 13-26.

Hołdyński Cz., 1991. Flora segetalna, zróżnicowanie florystyczno-ekologiczne i przemiany szaty roślinnej pól uprawnych w aktualnych warunkach agroekologicznych Żuław Wiślanych. / Segetal flora, floristic and ecological diversity and transformations of the plant cover of cultivated fields in current agro-ecological conditions of the Vistula Delta Plain. Acta Acad. Agricult. Tech. Olsz., Agricultura, 51 (403): 1-51.

Jędruszczak M., Antoszek R., 2002. Ocena wrażliwości Echinochloa crus-galli (L.) Beauw. Na atrazynę i metrabuzynę. / Assessment of sensitivity of Echinochloa crus-galli (L.) Beauw. to atrazine and metrabuzin. Pam. Puł. 129: 51-59.

Kapeluszny J., 1979. Zachwaszczenie upraw ziemniaka na niektórych glebach środkowo-wschodniej Polski. Cześć I. Zespoły chwastów. / Weed infestation of potato crops on some soils of central-eastern Poland. Part 1. Weed associations. Ann. UMCS. Sect. E, 34 (5): 49-61.

Kornaś J., 1950. Zespoły roślinne Jury Krakowskiej. Część I. Zespoły pól uprawnych. / Plant associations of the Kraków Jurassic area. Part 1. Associations of cultivated fields. Acta Soc. Bot. Pol. 20 (2): 361-438.

Kornaś J., 1972. Zespoły synantropijne. / Synantrophic associations. [In:] Szafer W., Zarzycki K. Szata roślinna Polski. / Poland's plant cover. I. PWN, Warszawa: 442463.

Korniak T., 1992. Ekspansywne gatunki chwastów segetalnych w północno-wschodniej części Polski. / Expansive species of segetal weeds in the north-eastern part of Poland. Zesz. Nauk. AR w Krakowie, 261 (33): 27-36. 
Kozak M., 2002: Zbiorowiska segetalne gminy Rudnik (województwo opolskie). / Segetal communities of the gmina (commune) of Rudnik (Opolskie Voivodship (region)). Fragm. Flor. Geobot. Polon. 9: 219-272.

Kutyna I., 1988. Zachwaszczenie roślin uprawnych oraz zbiorowiska segetalne zachodniej części Kotliny Gorzowskiej i terenów przyległych. / Weed infestation of crop plants and segetal communities of the western part of the Gorzów Valley and adjacent areas. AR w Szczecinie, Rozprawy, 116: 1-107.

Kuźniewski E., 1974. Interesujące zbiorowiska chwastów występujących w roślinach okopowych na Śląsku. / Interesting weed communities occurring in root crops in Silesia. Pam. Puł., Prace IUNG, 60: 11-127.

Kuźniewski E., 1996. Aktualne wtórne zachwaszczenie plantacji roślin okopowych na Śląsku Opolskim. / The present secondary weed infestation of root crop plantations in Opole Silesia. Mat XXI Kraj. Konf. Wyd. IUNG Puławy: 37-42.

Matuszkiewicz W., 2001. Przewodnik do oznaczania zbiorowisk roślinnych Polski. / Guide for identification of Poland's plant communities. Wyd. Nauk. PWN, Warszawa: 1-537.

Mirek Z., Piękoś-Mirkowa H., Zając A., Zając M., 2002. Flowering plants and pteridophytes of Poland - a checklist. W. Szafer Institute of Botany, Polish Academy of Sciences: 1-300.

Pawłowski B., 1972. Skład i budowa zbiorowisk roślinnych oraz metody ich badania. / The composition and structure of plant communities and methods for their investigation. (In:) Szata roślinna Polski. PWN 1: 237-268.

Rola J., Rola H., Kucharczyk A., 1989. Problem odporności chwastów na herbicydy w warunkach Polski. / The problem of resistance of weeds to herbicides in the conditions of Poland. Mat. 29. Sesji Nauk. IOR, Poznań, część I - referaty: 57-74.

Rzymowska Z., 1999. Zbiorowiska roślinne pól uprawnych Podlaskiego Przełomu Bugu. / Plant communities of cultivated fields of the Podlaski Przełom Bugu mesoregion. Manuskrypt rozpr. dokt.: 39-101.

Siciński J. T., 1974. Zbiorowiska segetalne Kotliny Szczercowskiej (Widawskiej). / Segetal communities of the Szczerowska (Widawska) Valley. Acta. Agrobot. 27 (2): 5-94.

Siciński J. T., Sowa R., Warcholińska A. U., Wiśniewski J., Wnuk Z., 1978. Zróżnicowanie florystyczno-ekologiczne zbiorowisk segetalnych w środkowej Polsce / Floristic and ecological diversity of segetal communities in central Poland. [In:] Sowa R., Warcholińska A.U. Niektóre aspekty biologii chwastów segetalnych. / Certain aspects of biology of segetal weeds. Mat II Kraj. Konf. Wyd. IUNG Puławy: 27- 42.

Siciński J. T., 2003. Agrofitocenozy dorzecza środkowej Warty i Bzury - stan, dynamika i zagrożenia. / Agrophytocenoses of the middle Warta and Bzura rivers basin - the state, dynamics and threats. Wyd. Uniw Łódz.: 1-55.
Skrzyczyńska J., 1994. Studia nad florą i zbiorowiskami segetalnymi Wysoczyzny Siedleckiej. / Studies on the flora and segetal communities of the Siedlce Upland. Rozpr. WSR-P w Siedlcach, 39: 5-145.

Skrzyczyńska J., Skrajna T., 2004. Roślinność pól Wysoczyzny Kałuszyńskiej. Cz. I Zespoły zbożowe. / Vegetation of fields of the Kałuszyńska Upland. Part 1. Cereal plant associations. Fragm. Agron. 4 (84): 32-44.

Skrajna T., Skrzyczyńska J., 2004. Zbiorowiska upraw okopowych gminy Grębków (woj. Mazowieckie). I Root crop communities of the gmina (commune) of Grębków (Mazowieckie Voivodship (region)). Zesz. Nauk. AP. Siedlce ser. Rolnictwo 65: 81-90.

Skrajna T., Skrzyczyńska J., 2005. Roślinność pól Wysoczyzny Kałuszyńskiej. Cz. II Zbiorowiska i zespoły ściernisk. / Vegetation of fields of the Kałuszyńska Upland. Part 2. Communities and associations of stubble fields. Fragm. Agron. 4 (88): 98-115.

Skrajna T., Skrzyczyńska J., 2006. Zbiorowiska zubożałe i kadłubowe Wysoczyzny Kałuszyńskiej. / Impoverished and species-poor communities of the Kałuszyńska Upland. Acta Sci. Pol., Biologia, 5 (1-2): 59-72.

Szotkowski P., 1981. Chwasty upraw okopowych i zbóż ozimych w południowo-wschodnim obszarze Śląska Opolskiego. / Weeds of root crops and winter cereals in the south-eastern area of Opole Silesia. OTPN. Wydział III Nauk Przyr. PWN, Warszawa-Wrocław, 190.

Szotkowski P., 1989. Zmiany we florze i zachwaszczeniu pól południowej części Śląska Opolskiego po 11 latach. / Changes in the flora and weed infestation of fields of the southern part of Opole Silesia after 11 years. OTPN. Zesz. Przyr.: 226.

Trąba Cz., Ziemińska M., 1994. Zbiorowiska chwastów na polach uprawnych w okolicach Zamościa. Cz. I. Zbiorowiska na rędzinach. / Weed communities in cultivated fields in the area of Zamość. Part 1. Communities on rendzina soils. Ann. UMCS, sect. E, 49 (14):89-98.

Warcholińska A. U., 1987. Zbiorowiska segetalne Wzgórz Radomszczańskich. / Segetal communities of the Radomszczańskie Hills. Bab. Fizjogr. Pol. Zach., Ser. B, 38: 123-153.

Warcholińska A. U., 1994. Zmiany roślinności segetalnej Równiny Piotrkowskiej w ostatnich 22 latach. Cz. II. Zbiorowiska chwastów upraw lnu i roślin okopowych. Changes in segetal vegetation of the Piotrków Plain over the last 22 years. Part 2 . Weed communities of flax crops and root plants. Acta Agrobot. 47 (1): 37-54.

Warcholińska A. U., 1998. Roślinność segetalna terenów rolniczych Puszczy Bolimowskiej i jej współczesne przemiany. / Segetal vegetation of agricultural areas of the Bolimow Forest and its contemporary transformations. Forest Acta Agrobot. 41 (2): 369-452.

Węgrzynek B., 2005. Roślinność segetalna Wyżyny Śląskiej. Cz. IV. Zbiorowiska chwastów upraw okopowych ze związku Panico-Setarion Siss. / Segetal vegetation of the Silesian Upland. Part 4. Weed communities of root crops in the alliance Panico-Setarion Siss. 1946. Natura Silesiae Superioris, 8: 39-53. 
Wnuk Z., 1976. Zbiorowiska chwastów segetalnych Pasma Przedborsko-Małogoskiego i przyległych terenów. Cz. I. Zbiorowiska upraw okopowych. / Segetal weed communities of the Przedborsko-Małogoskie Hills Range and adjacent areas. Part 1. Root crop communities. Zesz. Nauk. UŁ ser. II, 14: 85-122.

Wnuk Z., 1989. Zbiorowiska segetalne Wyżyny Częstochowskiej na tle zbiorowisk segetalnych Polski. / Segetal communities of the Częstochowa Upland against the background of Poland's segetal communities. Monogr. Bot. 71: 1-118.

Wnuk Z., Dymon E., Grzebyk D., 1989. Zbiorowiska segetalne Rzeszowa. / Segetal communities of Rzeszów. Zesz. Nauk. AR w Krakowie. Rolnictwo, 28: 76-90.

Wójcik Z., 1980. Plant communities of Mazovian cultivated fields. Part III: Root-crop communities. Pol. Ecol. Stud. 6 (3): 545-569.

Wójcik Z., 2000. Zbiorowiska segetalne Pojezierza Suwalskiego. / Segetal communities of the Suwałki Lake District. Fragm. Flor Geobot., ser. Polonica, 7: 167-208.

\section{Zbiorowiska i zespoły roślinne upraw okopowych Wysoczyzny Kałuszyńskiej}

\author{
Streszczenie
}

W pracy zawarto klasyfikację fitosocjologiczną i charakterystykę zbiorowisk wykształcających się w uprawach okopowych Wysoczyzny Kałuszyńskiej. Stwierdzono występowanie trzech zespołów: Digitarietum ischaemi, Echinochloo-Setarietum i LamioVeronicetum politae oraz dwóch zbiorowisk: Setaria pumila - Setaria viridis i zbiorowiska kadłubowego ze związku Panico-Setarion. Zróżnicowanie troficzne i wilgotnościowe siedlisk obrazują zbiorowiska zróżnicowane florystycznie, które zakwalifikowano do niższych jednostek syntaksonomicznych. W obrębie Digitarietum ischaemi wyróżniono dwa warianty, Echinochloo-Setarietum zróżnicowano na dwa podzespoły i sześć wariantów. 